\title{
NON-EXPLOSIVE MASS LOSS
}

\author{
N. J. WOOLF \\ University of Minnesota, Minn., U.S.A.
}

\begin{abstract}
The various mechanisms whereby stars might lose matter are considered, together with the observational evidence of the mass loss rates associated with these mechanisms. The results are shown in a diagram giving the fractional mass that a star loses by various mechanisms as a function of initial mass.
\end{abstract}

\section{Introduction}

Since the last major conference to discuss mass-loss (Hack, 1969), there has been an abrupt change in our appreciation of the role that red giant stars play in stellar evolution. Mass ejection has become clearly visible through infrared observations of recently condensed circumstellar dust (Gillett et al., 1968; Gehrz and Woolf, 1971), and through radio observation of molecules in these circumstellar clouds (Wilson and Barrett, 1972, Wilson et al., 1972, 1973). And indeed it has become clear to the observers that a substantial fraction of the matter contained in stars is returned to the interstellar medium by this process.

In this connection it is interesting that the interstellar gas seems very depleted in elements that would form solid condensates above about $1000 \mathrm{~K}$, but not those that would condense at cooler temperatures. The $\mathrm{Na} / \mathrm{Ca}$ problem of interstellar abundances that is a part of this has long been known. Unless most matter that returns to the interstellar medium can make solid condensates under circumstellar conditions, the interstellar gas abundances would not arise. Now moderately hot flows like those in planetary nebulae are known to be able to condense dust that did not arise in a previous red giant phase (Gillett et al., 1973). But the situation may well be different for supernova ejecta. If so, these interstellar matter observations would be stating that the mass flow from supernova explosions is only a small part of the total return of mass to the interstellar medium. And it justifies us concentrating here on nonexplosive mass loss.

This paper is divided into two main sections, each with sub-divisions. In the first we consider processes which might cause matter to be ejected, and decide which should be considered potentially important for stellar evolution. In the second section we consider the evolution of stars of various mass ranges, and attempt to infer when matter leaves them, and how much of the mass goes.

\section{Mass Ejection Mechanisms}

Some stars make a great display while they trickle off a little matter back to interstellar space. Others without much ado seem to return most of what originally formed them. Here we are attempting to find which phases have a mass ejection rate that significantly affects stellar evolution. It is also an attempt to find out which mechanisms operate the ejection process, so that their fuller implication for all areas of stellar evolution might be considered. 
Mass ejection significantly affects stellar evolution when the rate at which highest energy content fuel is depleted in the interior of a star is slower than the rate at which it is removed from the exterior. Thus if the exterior is hydrogen, ejection rates exceeding $1.4 \times 10^{-19} \mathrm{~g} \mathrm{erg}^{-1}$ radiated are significant. When the exterior is helium, ejection rates ten times larger are needed to be significant.

These rates should be considered in terms of the problem of a star reducing its mass enough to become a white dwarf, as appears to have happened to at least one star of the Pleiades (Luyten and Herbig, 1960). A $6 M_{\odot}$ star crosses the Hertzsprung gap with perhaps $20 \%$ of its original mass in a burned core. To avoid acquiring more than $1.4 M_{\odot}$ in its core, it will have to shed $\sim 4.6 M_{\odot}$, while only burning $\sim 0.2 M_{\odot}$. This must be a very efficient process.

\subsection{WAYS FOR A STAR TO REDUCE}

The possible means for a star to reduce its mass are:

(i) Use of radiation pressure to drive off upper atmospheric layers,

(ii) Feeding momentum into the outer layers through travelling waves,

(iii) Feeding energy into outer layers via turbulent or convective motions,

(iv) Release of energy that comes from the star being in a metastable state,

(v) Rotational shedding of matter at the equator by the star reducing its moment of inertia,

(vi) And giving the mass to a companion star.

Method (vi) is not helpful, since it only delays the day of reckoning. However it may be that binary stars can use their angular momentum to efficiently 'spill' mass while it is being transferred and this should be explored.

Method ( $v$ ) is believed to operate for Be stars, and has been suggested (Limber, 1964) to operate for WR stars. A discussion by Auer and Woolf (1965) shows that rotational shedding will not allow a star to lose a lot of mass. Stellar model predictions would have a Be star losing perhaps $0.5 \%$ of its mass, while observations may suggest ten times this amount. Neither amount is very significant.

Explosive mass ejection, (iv), does occur, and it has also been suggested that the hydrogen ionization and/or molecule formation energy of a red giant might lead to fast or slow dispersal of the outer layers. We shall consider mainly the observational evidence for slow mass loss, and the alternative schemes for causing it. We shall also consider the observed mass loss in planetary nebulae, which may be caused this way, but not supernova or nova explosions.

Thus we are left to consider, (i) radiation pressure driven winds, which have been discussed for red giants by Weymann (1962), Hoyle and Wickramasinghe (1962), and Gehrz and Woolf (1971).

Then there are travelling waves (ii) which should only be important for pulsating stars, and can so be observationally distinguished.* And there is (iii) mass loss via macroscopic motions, a process best seen in the solar corona.

* It is possible that on a small scale, travelling waves may propagate 'microtrubulence'; but we consider this under (iii). 


\subsection{EVIDENCE FOR MASS LOSS}

The evidence that mass loss does occur can be briefly summarized.

(1) White dwarfs are found at relics of stars at least $2.5 M_{\odot}$, and perhaps as much as $6 M_{\odot}$

(2) Stellar spectra show that envelopes are receding from some stars.

(3) Stars are found with extended optical, infrared or radio emitting envelopes surrounding them.

(4) There are some weak dynamical and stellar model indications that some more evolved stars now have less mass than less evolved stars in the same clusters (Caputo et al., 1973).

(5) The chemical composition of some stars seems to require that both mass loss and mixing have occurred. However this is always very difficult to distinguish from cases where mixing alone has happened to a highly evolved star.

Many of these phenomena are discussed in an article by Weymann (1963) and the book edited by Hack. Compositional changes are intriguing but tend to be inconclusive. Some rare low mass stars seem to develop almost pure helium envelopes (Hill, 1965). Nitrogen to carbon ratios seem highly variable, even in otherwise 'normal' blue stars (Walborn, 1971). And the red carbon stars seem to either be caused by a combination of normal mixing and mass loss (Thompson, 1972) or mixing via jets or plumes (Ulrich and Scalo, 1972) or both.

Some attempts have been made to total the fuel consumed by stars of various masses (see e.g. Hills and Dale, 1973). But for low mass stars, the fuel consumption is dominated by a short period of time spent as a high luminosity red giant, and this is hard to estimate observationally. One's best chance to pin down the lifetime of crucial phases are to use in addition to cluster membership, field c.p.m. pairs, total numbers of field stars of these types, and data on motions.

\subsection{RADIATIVE MASS LOSS}

If a star radiates a continuum, atoms in the outer atmosphere are in a reduced radiation environment because of the spectral lines absorbed by closer in atoms of the same kind, and even if the radiation field were present, the reduced excitation would leave only resonance lines potentially able to absorb momentum. If however the outer layers are disturbed, then the atoms may experience the full force exerted by the continuum on their resonance lines, in the Doppler line width from their disturbed motion. The momentum absorbed is at most $(L / c) \cdot(v / c)$, and it partially becomes momentum $v \mathrm{~d} m / \mathrm{d} t$ for outflowing matter with a mass loss rate $\mathrm{d} m / \mathrm{d} t$. Thus even if there are $N$ resonance lines, the maximum mass loss rate is given by:

$$
\underset{\mathrm{d} t_{\text {(line) }}}{\mathrm{d} M}<\frac{N L}{c^{2}} .
$$

If however a continuum can be used to absorb or scatter the radiation, this 
rate can be increased by a factor $c / N v$, giving a rate

$$
\underset{\mathrm{d} t \text { (continuum) }}{\mathrm{d} M}<\frac{L}{c v} .
$$

where $v$ is the outflow velocity.

But now the radiation field may be able to couple momentum across the envelope, and (2) is no longer a true limit. But in practice, considering plausible models one infers that (2) is unlikely to be in error by more that a factor $\sim 10$.

The line radiation process has been assumed to the responsible for mass ejection in early type supergiants (Morton, 1969). For such stars, even if $N=10$, one finds a rate of only $10^{-20} \mathrm{~g} \mathrm{erg}^{-1}$ which does not affect stellar evolution.

For late type stars, envelopes have long been known (e.g. Deutsch, 1956), and for these stars solid particles that form in the upper atmosphere can create continuous absorption and/or scattering. The envelopes are seen to expand at typical velocities of $15 \mathrm{~km} \mathrm{~s}^{-1}$, giving a possible mass loss rate from (2) of $\sim 2 \times 10^{-17} \mathrm{~g} \mathrm{erg}^{-1}$. If a star is in a suitable state to create enough solids for a long enough time, such a rate can dominate the future course of evolution.

\subsection{TRAVELling WAVES}

Since Schwarzschild's (1938) study of $\delta$ Cephei, it has seemed that oscillating stars might feed an appreciable fraction of their energy of oscillation into travelling waves. This might then possibly lift the outer layers of the star and eject them. Such ejection could be one of the means of damping the oscillations. At present it seems best to explore this possibility observationally. We know that solid particles seem able to form remarkably easily, and therefore one of the best tracers of mass ejection is infrared emission.

In 1970 Gehrz and Strecker found that AC Her, an RV Tauri star had a huge excess emission, and so Gehrz and Woolf (1970) observed other pulsating stars to see if this was a common phenomenon. We found that the rare RV Tauri stars do indeed have enormous excess emission, and Gehrz in his thesis estimated mass ejection rates of $5 \times 10^{-6} M_{\odot} \mathrm{yr}^{-1}$ to $6 \times 10^{-5} M_{\odot} \mathrm{yr}^{-1}$. But other pulsating stars showed little or no effect.

To be sure, Mira and SRa variables have envelopes around them, but their infrared radiation from their envelopes seemed to correlate with spectral type rather than with pulsation amplitude. And for this reason, and also the comparison of mass ejection rate to luminosity, we believe the radiation pressure effect dominates there. For these stars the typical ejection rate is about $4 \times 10^{-18} \mathrm{~g} \mathrm{erg}^{-1}$, a factor smaller than given by (2).

Small infrared excesses were found for the longest period cepheids, SV Vul and RS Pup $\left(M_{v} \sim-6\right)$, and the second of these has the outer parts of its shell visible (Havlen, 1971). From the infrared excess, the inferred current mass ejection rate is $\sim 10^{-6} M_{\odot} \mathrm{yr}^{-1}$, however the nebula density distribution and density seem to refer to a 10 to 100 times larger mass ejection rate over the past $10^{5}$ to $10^{4} \mathrm{yr}$. Possibly 
this refers to a preceding red giant phase. The current rate of mass ejection would not be significant over the limited lifetime as a cepheid.

For very high mass stars, perhaps above $60 M_{\odot}$, pulsational mass loss may also occur. Here there is a possible reason for the dense infrared envelopes around $\eta$ Car and CIT +10420 , and perhaps the shell around P Cygni where the mass ejection rate seems to be $\sim 10^{-4} M_{\odot} \mathrm{yr}^{-1}$. Even for a $100 M_{\odot}$ star, living a few times $10^{6} \mathrm{yr}$, such rates are decisive. But the total lifetime of such phases needs investigation. Some of the most massive stars may spend their lives shrouded in dusty shells that they are ejecting (Hoyle et al., 1973) and they may never be seen as the luminous stars that they really are.

For W Virginis stars there is good spectroscopic evidence for believing that matter is ejected, but the infrared observations are not yet good enough to give the rate. These stars are probably too rare to be important as major sites of mass ejection.

\subsection{Convective MASS EJECTION}

This process is well known in the Sun, where convected energy heats up the corona to such a temperature that the particles have escape velocity from their thermal energy. It is possible that the ejection rates in lower main sequence stars might explain their lithium depletion (Weymann and Sears, 1964), but total mass depletion is insignificant.

One might attempt to theoretically estimate rates of mass ejection by this process, as has De Jager (1959) who decided it was not significant. Or we can use some kind of observations to imply a limit to it as did Weymann (1963). And we should expect that the more luminous the star, the greater the mass flow might be. Now return again to the long period cepheids. Stars of similar luminosity and temperature but non variable do not show excess infrared emission. Suppose that they all have coronae due to convection. Now if the coronal mass ejection is larger than the pulsational mass ejection, the pulsational mass ejection will be absorbed into the main mass flow, and there should be no infrared excess. Since we observe the infrared excess it seems likely that the convective mass loss is smaller than $10^{-6} M_{\odot} \mathrm{yr}^{-1}$, and as such it seems unlikely to be very important.

Again for the most luminous $\mathrm{G}$ and $\mathrm{K}$ supergiants there seems to be a mass ejection process that causes dust to form (Humphreys et al., 1971). And since it does not obviously refer to a pulsation driven process, one wonders whether it might be this mechanism. However as one examines cool stars of higher and higher luminosity, one finds that there appears to be a continuous sequence linking all these stars ejecting mass. It is clearly seen at earliest types M6III, M5II, M1Iab, G8Ia and $\mathrm{GOIa}^{+}$. Thus it seems to all be part of a radiation pressure driven process.

One major problem for stellar wind mass loss is that one initially predicts that it would be most effective for red giant stars. But these stars condense dust that acts as a coolant for the outer layers, while simultaneously activating its own mass loss process. 


\section{Ejection for Stars of Different Mass Ranges}

\subsection{StaRs Below $2 M_{\odot}$}

For these stars there is clear evidence that red giant mass loss is crucial. We shall be concerned with determining the mass loss rates and the duration of its operation. The stars at the tip of the red giant branches are radiating about $2 \times 10^{37} \mathrm{erg} \mathrm{s}^{-1}$. And using inequality (2), we find a maximum ejection rate could be $6 \times 10^{-6} M_{\odot} \mathrm{yr}^{-1}$ for an ejection velocity of $15 \mathrm{~km} \mathrm{~s}^{-1}$. We shall infer somewhat smaller rates from the observations.

Tsuji (1971) has attempted to use optical observations to derive mass ejection rates for two $\mathrm{S}$ type Mira stars, $\mathrm{R}$ Cyg and $\mathrm{R}$ And. He found the rate would be $3 \times 10^{-8} M_{\odot} \mathrm{yr}^{-1}$ if all the matter were neutral. But this had to be increased by a large but very uncertain factor to allow for ionization in the envelope. His final estimate was a rate between $7 \times 10^{-6}$ and $1.5 \times 10^{-5} M_{\odot} \mathrm{yr}^{-1}$.

R Cyg has been observed from 3-11 $\mu$ in the infrared by Gillett et al. (1971), and it has infrared excess emission which is entirely typical of other giant stars of similar temperature. An $11 \mu$ peak emission and another one near $18 \mu$ are attributed to emission by silicate particles formed in the envelope, and from this we can obtain a second estimate of mass ejection rates. We assume that all matter that might condense as silicates ( $1 / 250$ by mass for a normal composition mixture) does in fact condense. The distance where the silicates condense is estimated from effects of optical depth on the silicate emission bands. The amount of matter is estimated from the silicate band strength, and the ejection velocities are taken from optical and radio data. By this means Gehrz and Woolf (1971) estimated ejection rates for typical disc and halo long period variables (Mira and SRa) to be about $1.4 \times 10^{-6} M_{\odot} \mathrm{yr}^{-1}$.

Alternative ways of calculating mass ejection rates come from the observation of $\mathrm{OH}$ and $\mathrm{H}_{2} \mathrm{O}$ masers in the envelopes of some stars. Here there may be some dispute about the physical conditions that give rise to masers. None the less, the rates calculated this way also seem to be about $10^{-6} M_{\odot} \mathrm{yr}^{-1}$. For all these stars the rates calculated depend upon the surface area of the star. Now a crude theoretical estimate for red giant stars would have that at a given surface temperature, the luminosity and hence surface area is proportional to the mass. Thus since the masses of these stars only vary by perhaps a factor 3 , their sizes must be similar, and the use of a single rate to describe all the stars may be appropriate.

The only LPVs for which both lifetimes and mass ejection rates can be estimated are the 150-200 day Miras which are found in some globular clusters. In a study of such stars in the field, T. J. Pepin and I (unpublished) found that these stars also had excess infrared emission, which seemed to come from closer in denser shells. And when we had corrected for a heavy element deficiency of a factor 3 , it seemed that the matter ejection rates were similar to those for the more usual longer period Miras and SRa stars. For the rich cluster 47 Tucanae there are three Mira stars, and I estimate that there is about 1 evolved star per $5 \times 10^{4} \mathrm{yr}$ of a given phase. Thus these Mira stars may only last about $1.5 \times 10^{5} \mathrm{yr}$, ejecting only about $0.2 M_{\odot}$ while 
they do so. However for such stars there is little mass expected to be available between an initial mass of perhaps $0.9 M_{\odot}$, and a final white dwarf of perhaps $0.6 M_{\odot}$.

Other estimates for typical Miras suggest that they last far longer. Thus at high galactic latitudes there are about 30 Mira stars for every dense planetary nebula. The expansion lifetime of such a planetary nebula is about $2 \times 10^{4} \mathrm{yr}$, giving the Mira stars about $6 \times 10^{5} \mathrm{yr}$ each under the assumption that all the low mass stars go through both phases. The average Mira stars would be ejecting about $0.8 M_{\odot}$ each. Regardless of the precise figures it is hard to avoid the conclusion that red giant mass-loss is more important than planetary nebula mass-loss.

Alternatively we can estimate the total mass ejection from stars that populate high latitudes. I have used infrared absolute magnitudes (which should be more constant than visual absolute magnitudes) to estimate the space density and scale height of the LPV's seen at high latitudes. I find a scale height of $600 \mathrm{pc}$, and a local space density of $8.0 \times 10^{-8}$ stars $\mathrm{pc}^{-3}$. Then the total matter returned to the galactic plane from these stars is $1.4 \times 10^{-10} M_{\odot} \mathrm{pc}^{-2} \mathrm{yr}^{-1}$. Deutsch (1969) has estimated that stars of less than $2 M_{\odot}$ eject $2 \times 10^{-10} M_{\odot} \mathrm{pc}^{-2} \mathrm{yr}^{-1}$. The near coincidence of the two rates suggests little need to search for further mass ejection phases for these stars. To our estimate for matter from LPV's, we should perhaps add a further $20 \%$ to allow for planetary nebulae, and another $10 \%$ to allow for mass ejection while the star is a somewhat warmer red giant than the LPV's.

For Population II stars, because of the reduced heavy element abundance, radiation pressure driven mass loss will be less effective. Indeed it seems that when the heavy element abundance is reduced by more than a factor of about 10 , the process should abruptly stop because of the star's gravitational field. This has the consequence that we may find an abrupt change in the evolution of globular cluster stars at about this chemical composition. There have been observational indications of globular cluster star mass loss for M3 (Woolf, 1964) but not M13 (King, 1962). And one wonders whether the above process might not cause the difference. In M3 the coolest star does show TiO bands. It is an SRd variable that may be a Population II extension of the LPV's. Gehrz in his thesis found that stars of this type such as SX Her did indeed seem to be ejecting matter.

Population II mass loss cutting off have important consequences for the evolution of our Galaxy. If mass loss did not occur, then many more stars should have become supernovae, and the heavy element enrichment of the Galaxy could have been speeded up (Gilman, private communication).

\subsection{StaRs OF $2 M_{\odot}$ TO $6 M_{\odot}$}

If stars in this mass range do become white dwarfs, they must lose between $50 \%$ and $80 \%$ of their initial mass. Questions arise (a) can we show that mass loss has occurred on this vast scale and (b) can we find in which phases it occurred?

Let us start by adopting a common current assumption, that the nuclei of planetary nebulae are hot white dwarf stars with a thin fringe of non degenerate matter around them. Now planetary nebulae are known with A or B type companions. For NGC 3132 
the companion appears to be an A3V star (Brown et al., 1970), and the combined planetary nebula and carbon star UV Aur also has a B8.5 companion. These cases seem to be different from NGC 1514 which seems to have a horizontal branch star companion (Greenstein, 1973): Then these planetary nebulae imply that main sequence late B stars have been able to form white dwarfs, and the white dwarf suspected Pleiad is also consistent with this. Thus it seems that mass loss has occurred on this vast scale.

Now not only UV Aur, but other carbon stars too have known A or B type companions, so that some of these must also have been formed in this mass range. Indeed one carbon star appears to have a B5 companion. Also the distribution of long period $\mathrm{S}$ stars would be consistent with many of them too coming from these stars. In the CIT $2 \mu$ survey there are many 'infrared stars', very cool stars that are mainly $\mathbf{M}$ stars, but some are $\mathrm{C}$ stars. These have a flattened distribution. And in the original attempt to identify the source of radiation, a number of these were misidentified as nearby A and B stars. This suggests that some of the A and B stars may well be physical companions. Thus there seem to be red giants that might be sites of mass ejection. Unfortunately there seems no way of estimating whether there are enough red giants. A check of cool stars for membership of galactic clusters in the right mass range could give an answer.

The more massive stars of this group form short period cepheid variables, but these do not seem to be ejecting much matter. Equally, although most of these stars rotate rapidly, it seems unlikely that rotational mass ejection is important. We are left with the conclusion that mass ejection is important for these stars, but without further information we cannot partition the responsibility between planetary nebulae and red giants.

\subsection{Stars of $6 M_{\odot}$ to $20 M_{\odot}$}

A typical group of stars of this kind might be the Per I association. There are here $22 \mathrm{M}$ supergiants of class Iab, but there are no known S, C or infrared stars that might belong to this association. (Though again we must remember $\mathrm{W} \mathrm{CMa}$ and its B5 companion.) The estimated initial mass is about $15 M_{\odot}$. And for such stars the lifetime as a red giant is 5 or possibly $15 \times 10^{5} \mathrm{yr}$ (Barbaro et al., 1971), depending on the importance of neutrino processes.

Now the mass ejection rate of the Per I stars has only been observed for a few stars in that association, but for the other stars, comparable objects have been observed elsewhere, and we can crudely estimate the total mass ejection. About $10 \%$ of the time as a red giant is spent with a rate $\sim 3 \times 10^{-5} M_{\odot} \mathrm{yr}^{-1}$, about $30 \%$ is spent with a rate $\sim 5 \times 10^{-6} M_{\odot} \mathrm{yr}^{-1}$, and the remaining $60 \%$ is with a rate $\sim 10^{-6} M_{\odot} \mathrm{yr}^{-1}$. The total ejection is in the range 2.5 to $7.5 M_{\odot}$, with a preference for the smaller figure.

Stars in this mass range will have a small rotational mass loss near the main sequence and possibly a small loss as a cepheid, but there are no other phases at which substantial mass loss is expected. Of course there could be phases with mass 
ejection rates $\sim 10^{-4} M_{\odot} \mathrm{yr}^{-1}$, which last too short a time to find even one star, but even then it seems likely that we will have to conclude that stars in this mass range do not manage to shed more than perhaps $50 \%$ of their initial mass before some catastrophic phenomenon removes them from the HR diagram. The coming into importance of neutrino energy loss process in this mass range could make a crucial difference: neutrino pressure cannot sustain mass loss at low densities, whereas radiation pressure can. The mass ejection from $S$ Persei is about $4 \times 10^{-18} \mathrm{~g} \mathrm{erg}^{-1}$ radiated, but if the neutrino luminosity is several times the radiative luminosity, the mass loss rate may not be able to affect the evolution.

\subsection{Stars ABove $20 M_{\odot}$}

As stars become more massive, and radiation pressure dominates their structure, it becomes possible to lose mass before the interior becomes hot and neutrino processes take over. In this mass range we find Of and WR stars, there are O-B supergiants that are probably using line radiation pressure to eject matter (Morton, 1969), at higher luminosities there are stars like P Cygni, there are $\mathrm{G}$ and $\mathrm{K}$ supergiants with dusty envelopes, and there are some class Ia $\mathrm{M}$ stars, all busy ejecting matter.

But in this rather wide range of masses there is no easy way to discriminate between different initial mass stars. Luminosity is nearly proportional to mass, and evolutionary time scales are almost independent of it. With observed mass loss rates it would be surprising if the most massive stars of this group do not eject most of their original mass before becoming a supernova. For the least massive stars of this group, the conclusions for the 6-20 $M_{\odot}$ stars probably apply. It does not seem possible to estimate the mass where a transition occurs.

\section{Conclusions}

The mass loss processes that seem to affect stellar evolution are, radiation pressure driven, pulsation driven and single events caused by metastable states. In practice these seem to come down to radiation pressure driven mass loss for red giants, pulsation driven mass loss for the most massive and luminous stars and formation of planetary nebulae or supernovae. Other processes may be important for rare stars.

The oldest lowest mass stars lose relatively little. and indeed for stars with less than $10 \%$ of the solar heavy element abundance, the red giant mass loss process fails. For stars with more nearly Population I composition, there is a mass loss of $\sim 0.2 M_{\odot}$ in the red giant phase at a rate $\sim 1.4 \times 10^{-6} M_{\odot} \mathrm{yr}^{-1}$.

Somewhat more massive stars up to $2 M_{\odot}$ appear to have increasing red giant mass ejection, with the phase lasting longer as more matter needs to be ejected. For these stars the red giant phase ejects typically $5 \times$ the matter lost as a planetary nebula. Up to $6 M_{\odot}$, it appears that stars still succeed in becoming white dwarfs, but the appropriate red giant statistics do not yet exist to demonstrate whether in this mass range the red giants or planetary nebulae are the most important sites of mass loss.

For stars of $6-20 M_{\odot}$, it appears that red giant loss, the dominant process of 
ejection fails to reduce the initial mass by more than $\sim 50 \%$. And the star probably becomes a supernova while still having a substantial mass outside the core. For somewhat higher masses the star seems to be losing matter at a high rate most of the time. For stars above some high mass it seems likely that most of the matter is returned to the interstellar medium before the core implodes.

In Figure 1 there has been an attempt to schematically indicate the mass ejection

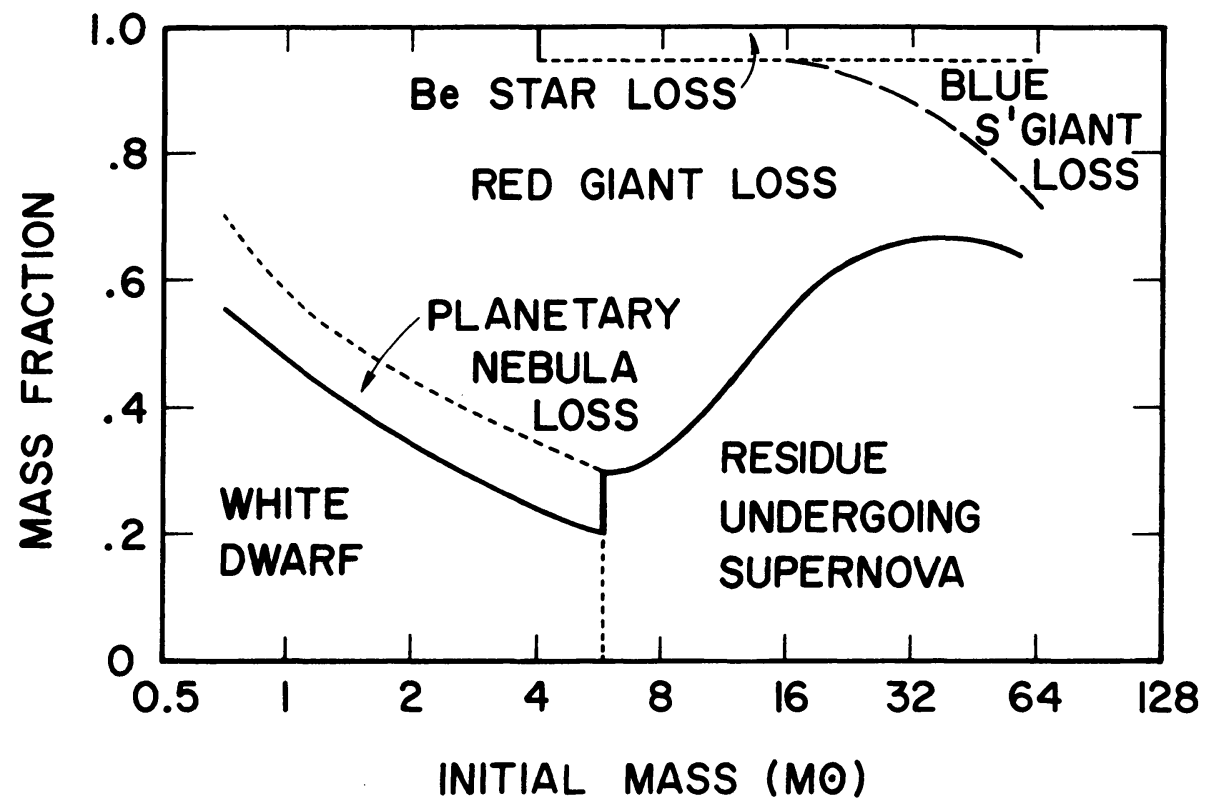

Fig. 1. Mass ejection prior to a supernova (if that should happen) as a function of mass. Although the diagram is based on observations, the interpretation is both speculative and personal.

as a function of initial mass. The uncertainties about total mass loss increase above $6 M_{\odot}$, and the diagram for this portion indicates mainly that even in this mass range, ejection cannot continue to be ignored.

\section{Acknowledgement}

This research was supported by N.S.F. under grant GP32772.

\section{References}

Auer, L. H. and Woolf, N. J.: 1965, Astrophys. J. 142, 182.

Barbaro, G., Chiosi, C., and Nobili, L. : in M. Hack (ed.), Colloquium on Supergiant Stars, Trieste Obs. Brown, S., Higginbotham, N., and Lee, P.: 1970, Publ. Astron. Soc. Pacific 82, 1372.

Caputo, F., Nata, A., and Castellani, V.: 1973, Astrophys. Space Sci. 22, 213.

De Jager, C.: 1959, in 9th Colloque Int. d'Astrophysique, Institut D'Astrophysique, Liège, p. 280.

Deutsch, A. J.: 1956, Astrophys. J. 123, 210.

Deutsch, A. J.: 1969, See Hack 1969, p. 1. 
Gehrz, R. D. and Woolf, N. J.: 1970, Astrophys. J. 161, L213.

Gehrz, R. D. and Woolf, N. J.: 1971, Astrophys. J. 165, 285.

Gillett, F. C., Low, F. J., and Stein, W. A.: 1968, Astrophys. J. 154, 677.

Gillett, F. C., Merrill, K. M., and Stein, W. A.: 1971, Astrophys. J. 164, 83.

Gillett, F. C., Forrest, W. J., and Merrill, K. M.: 1973, Astrophys. J. 183, 87.

Greenstein, J. L.: 1973, Astrophys. J. 173, 367.

Hack, M. (ed.): 1969, Mass Loss From Stars, D. Reidel Publ. Co., Dordrecht Holland.

Havlen, R. J.: 1971, Astron. Astrophys. 16, 252.

Hill, P. W.: 1965, Monthly Notices Roy. Astron. Soc. 129. 137.

Hills, R. J. and Dale, T. M.: 1973, Astrophys. J. 185, 937.

Hoyle, F., Solomon, P. M., and Woolf, N. J.: 1973, Astrophys. J. Letters 185, L89.

Hoyle, F. and Wickramasinghe, N. C.: 1962, Monthly Notices Roy. Astron. Soc. 124, 417.

Humphreys, R. M., Strecker, D. W., and Ney, E. P.: 1971, Astrophys. J. 167, L35.

King, I. R.: 1962, Astrophys. J. 136, 784.

Limber, D. N.: 1964, Astrophys. J. 139, 1251.

Luyten, W and Herbig, G. H.: 1960, Harvard Announcement Card, 1474.

Morton, D. C. : 1969, in M. Hack (ed.), Mass Loss From Stars, D. Reidel Publ. Co., Dordrecht, p. 36.

Schwarzschild, M.: 1938, Harvard Circ. No. 431.

Thompson, R. I.: 1972, Astrophys. J 172, 391.

Tsuji, T.: 1971, Publ. Astron. Soc. Japan 23, 275.

Ulrich, R. K. and Scalo, J. M.: 1972, Astrophys. J. 176, L37.

Walborn, N. R.: 1971, Astrophys. J. 165, L67.

Weymann, R. J.: 1962, Astrophys. J. 136, 476.

Weymann, R. J.: 1963, Ann. Rev. Astron. Astrophys. 1, 97.

Weymann, R. J. and Sears, R. L.: 1965, Astrophys. J. 142, 174.

Wilson, W. J. and Barrett, A. H.: 1972, Astron. Astrophys. 17, 385.

Wilson, W. J., Schwartz, P. R., and Epstein, E. E.: 1973, Astrophys. J. 183, 871.

Wilson, W. J., Schwartz, P. R., Neugebauer, G., Harvey, P. M., and Becklin, E. E.: 1972, Astrophys. J. $177,523$.

Woolf, N. J.: 1964, Astrophys. J. 139, 1081. 